\title{
Efficacy and safety of tofacitinib in the treatment of rheumatoid arthritis: a systematic review and meta-analysis
}

Ying $\mathrm{He}^{\dagger}$, Angel YS Wong ${ }^{\dagger}$, Esther W Chan ${ }^{*}$, Wallis CY Lau, Kenneth KC Man, Celine SL Chui, Alan J Worsley and lan CK Wong

\begin{abstract}
Background: Tofacitinib is a disease-modifying antirheumatic drug (DMARD) which was recently approved by US Food and Drug Administration (FDA). There are several randomised clinical trials (RCTs) that have investigated the efficacy and safety of tofacitinib in adult patients with rheumatoid arthritis (RA). A systematic review with a metaanalysis of RCTs was undertaken to determine the efficacy and safety of tofacitinib in treating patients with RA.

Methods: Electronic and clinical trials register databases were searched for published RCTs of tofacitinib between 2009 and 2013. Outcomes of interest include 20\% and 50\% improvement in the American College of Rheumatology Scale (ACR20 and ACR50) response rates, rates of infection, the number of immunological/ haematological adverse events (AEs), deranged laboratory results (hepatic, renal, haematological tests and lipoprotein level) and the incidence of drug withdrawal.
\end{abstract}

Results: Eight RCTs ( $n=3,791)$ were reviewed. Significantly greater ACR20 response rates were observed in patients receiving tofacitinib 5 and $10 \mathrm{mg}$ bid (twice daily) versus placebo at week 12, with risk ratios (RR) of 2.20 (95\% Cl $1.58,3.07)$ and $2.38(95 \% \mathrm{Cl} 1.81,3.14)$ respectively. The effect was maintained at week 24 for $5 \mathrm{mg}$ bid (RR 1.94; $95 \% \mathrm{Cl} 1.55,2.44)$ and $10 \mathrm{mg}$ bid (RR 2.20; $95 \% \mathrm{Cl} 1.76,2.75)$. The ACR50 response rate was also significantly higher for patients receiving tofacitinib $5 \mathrm{mg}$ bid (RR 2.91; 95\% Cl 2.03, 4.16) and $10 \mathrm{mg}$ bid (RR 3.32; $95 \% \mathrm{Cl} 2.33$, 4.72) compared to placebo at week 12. Patients in the tofacitinib group had significantly lower mean neutrophil counts, higher serum creatinine, higher percentage change of LDL/HDL and a higher risk of ALT/AST > 1 ULN (upper limit of normal) versus placebo. There were no significant differences in AEs and withdrawal due to AEs compared to placebo.

Conclusion: Tofacitinib is efficacious and well tolerated in patients with MTX-resistant RA up to a period of 24 weeks. However, haematological, liver function tests and lipoproteins should be monitored. Long-term efficacy and pharmacovigilance studies are recommended.

Keywords: Tofacitinib, Rheumatoid arthritis, Treatment outcome, Meta-analysis

\footnotetext{
* Correspondence: ewchan@hku.hk

'Equal contributors

Centre for Safe Medication Practice and Research, Department of

Pharmacology and Pharmacy, The University of Hong Kong, Pokfulam, Hong

Kong
} 


\section{Background}

Rheumatoid arthritis (RA) is a chronic inflammatory arthritis and a systemic autoimmune disease, which can lead to long-term joint damage, loss of function and disability [1]. In addition to the symptoms of joint destruction such as pain, swelling, or stiffness of the joints, those patients with RA may also suffer from other extra-articular manifestations, namely rheumatoid nodules [2], interstitial lung disease [3], cardiac involvement [4], and Felty's syndrome [5]. The onset of RA typically occurs between 30 to 50 years age. In the United Kingdom, the highest incidence is observed in people over 70 years of age [6]. It is estimated that the adult prevalence of RA is $0.5-1 \%$ in Europe and $1 \%$ in the United States $[1,7,8]$. Within two years of onset, approximately one third of people with RA are unable to continue with employment due to the disease [6]. The mortality rate of those people with RA is approximately twice of those without [9].

Guidelines for the management of RA have been issued by the American College of Rheumatology (ACR) [7,10], and by the National Institute for Health and Clinical Excellence guidelines in 2009 (NICE CG79) [6]. It is suggested that there are three main steps in the management of RA: pharmacological, non-pharmacological and surgical treatment $[6,7]$.

Pharmacological treatment of RA generally includes: non-steroidal anti-inflammatory drugs (NSAIDs), glucocorticoid treatment, disease-modifying antirheumatic drugs (DMARDs), and biologic agents $[7,10]$. By treating inflammation, relieving pain and/or suppressing the immune response, NSAIDs and glucocorticoids are able to alleviate some of the symptoms associated with RA. Currently, DMARDs such as methotrexate (MTX) remain the main treatment approach [7]. Antirheumatic biologics, including the tumor necrosis factor (TNF) inhibitors such as adalimumab or non-TNF inhibitors [10] are usually considered when the other treatment approaches are not sufficiently effective. There are still about one third of patients who have an unsatisfactory response to available treatments [11]. Consequently, development of new drugs and therapy for RA is needed.

Tofacitinib (CP-690550), also called tasocitinib [12] during early development with the commercial name Xeljanz ${ }^{\circ}$, is a new oral DMARD and an alternative to biologics. It was approved by the U.S. Food and Drug Administration (FDA) on 6 November 2012 [13]. It is a Janus Kinase (JAK) inhibitor which primarily inhibits JAK1 and 3, with a reduced inhibition of JAK2 [14]. It is suggested that as a JAK1/3 inhibitor, tofacitinib might suppress cytokine/chemokine expression and the immune activation through the JAK/STAT interferon-dependent signaling pathway, thus offering a promising target for the treatment of RA [15-17].

Tofacitinib is licensed to treat adult patients with active RA in the United States, especially for those either unable to tolerate MTX or biological therapies or who have an inadequate response. During the development of tofacitinib, a series of phase II [18-21] and phase III [22-25] clinical trials were conducted in adult patients at multiple treatment centres.

To our knowledge, no systematic review has been published to evaluate the efficacy and associated AEs of tofacitinib in the treatment of RA. In this study, we undertook a systematic review with a meta-analysis of randomised controlled trials (RCTs) to investigate the efficacy and safety of tofacitinib in treating patients with RA.

Our primary objective is to compare the response rates [20\% and 50\% improvement in the ACR scale (ACR20) and (ACR50)] of patients receiving tofacitinib versus placebo or adalimumab. The secondary objectives are i) to compare the incidence of infections, immunological or haematological AEs in those patients receiving tofacitinib versus placebo; ii) to compare the laboratory findings in those patients receiving tofacitinib versus placebo; and iii) to compare the incidence of withdrawal from the trials in those patients receiving tofacitinib versus placebo.

\section{Methods}

We performed this systematic review in accordance with the Preferred Reporting Items for Systematic Reviews and Meta-Analyses (PRISMA) Statement [26]. We searched EMBASE, the Cochrane Library and PubMed using keywords as follows: (tofacitinib) OR (tasocitinib) OR (CP-690, 550). Trial registers: the metaRegister of Controlled Trials (www.controlled-trials.com), the Clinical trials government (ClinicalTrials.gov) and World Health Organization International Clinical Trials Registry Platform (ICTRP) (http:// www.who.int/ictrp/en/) were also searched to identify potentially relevant studies. All databases were searched on March 1, 2013. Titles, abstracts and the content of the articles were screened to determine whether the articles met the inclusion criteria. Reference lists from retrieved studies were reviewed for the identification of potentially relevant studies. The search result was presented in Figure 1.

\section{Inclusion criteria}

The inclusion criteria for this systematic review were those published RCTs investigating the efficacy and safety of tofacitinib in adult patients (aged 18 or above) who had a diagnosis of active RA defined according to the ACR 1987 revised criteria for RA [27]. The exclusion criteria were conference proceedings as we were unable to assess the quality of these studies. Studies examining drug treatments other than tofacitinib were also excluded. Studies that did not report the primary outcomes (ACR20/50 response rates) were also excluded. Further evaluation on the full text was conducted for inclusion assessment. 


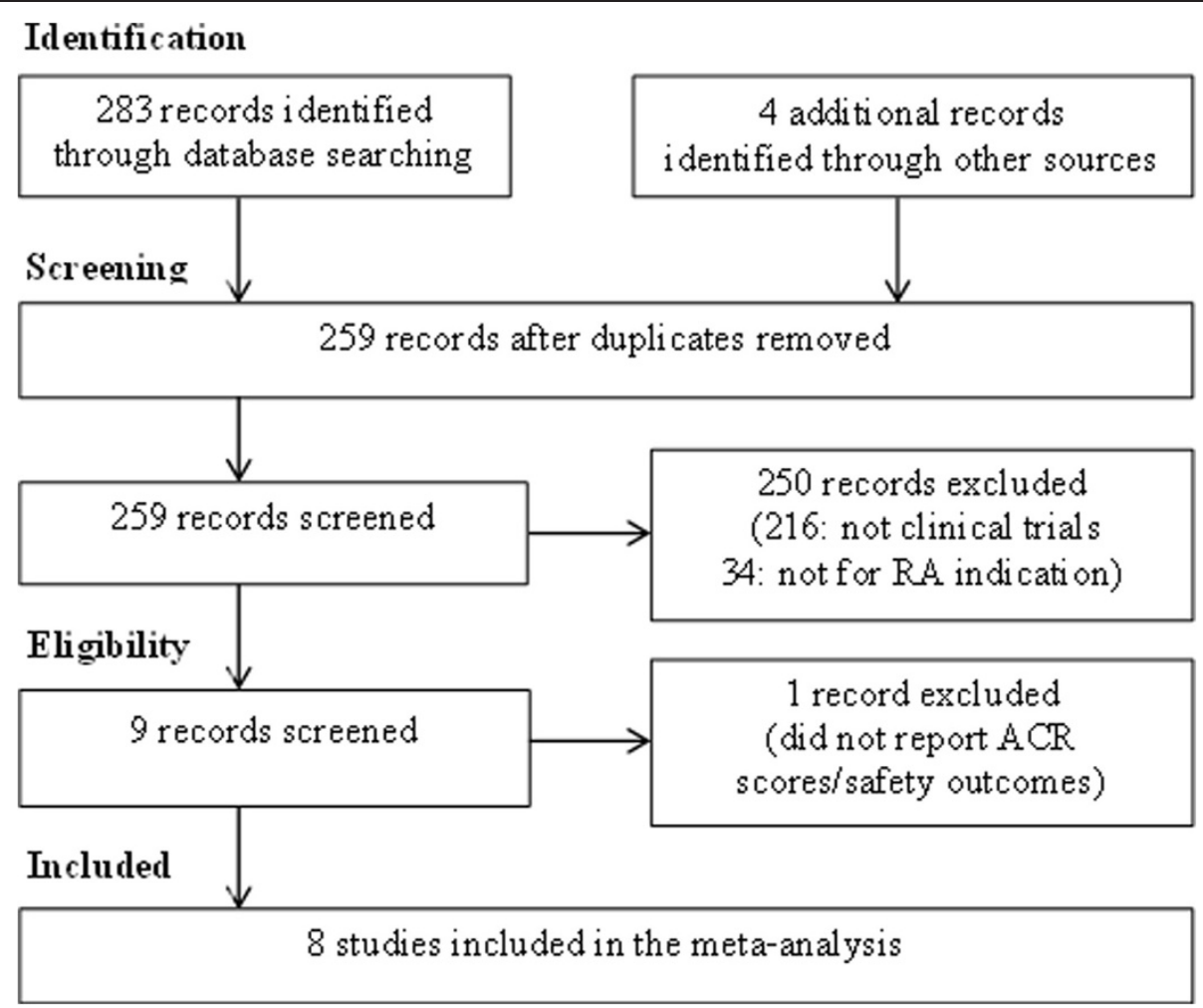

Figure 1 Review flowchart (PRISMA flowchart).

\section{Outcome measures}

The standardised response measurements for RA clinical trials of antirheumatic drugs, the ACR20 response rate and ACR50 response rate were selected as the primary outcome measures $[8,28]$.

Secondary outcomes included the number of patients with infections, immunological or haematological AEs, such as bronchitis, influenza, nasopharyngitis, pharyngitis, rash, upper respiratory tract infection, urinary tract infection and neutropenia. Laboratory parameters examined included the least squares mean changes in neutrophil count, haemoglobin levels and serum creatinine levels, incidence of alanine aminotransferase (ALT) and aspartate aminotransferase (AST) more than one times upper limit of the normal range (ULN), mean percentage changes of low-density lipoprotein (LDL) and highdensity lipoprotein (HDL). The incidence of patient withdrawal from treatment was also a secondary outcome of interest.

\section{Data extraction}

We performed the initial electronic database searches and screened the published abstracts for eligibility. Full texts were retrieved for potentially useful articles and then screened for relevance. Those relevant articles were then assessed independently by two reviewers for inclusion in the meta-analysis.
The data relating to the primary and secondary outcomes in all included studies were extracted by two independent reviewers and cross-checked by an additional reviewer for data accuracy. Non-statistical data extracted from the eligible studies included author, study setting, study duration, doses of tofacitinib, possible concomitant medication, sample size, mean scores on 3-variable Disease Activity Score in 28 joints (DAS28-3) using Creactive protein, mean scores on 4-variables (DAS28-4) using the erythrocyte sedimentation rate, mean scores on the Health Assessment Questionnaire disability index (HAQ-DI), and mean number of swollen joints and tender joints. Statistical data on ACR20 and ACR50 response rates, AEs and number of patient withdrawals were also extracted.

\section{Methodological quality assessment}

The risk of bias of the identified RCT articles was assessed using the Cochrane Collaboration's tool [29] (Additional file 1: Table S1). Assessment was conducted independently and cross-checked by additional reviewers with discrepancies resolved by consensus.

\section{Statistical analysis}

Risk ratios (RR) and mean differences were calculated for dichotomous and continuous outcomes respectively (e.g. ACR response rates, AEs and laboratory findings). 
The DerSimonian and Laird random-effects model was used to deal with possible heterogeneity between studies [30]. $\mathrm{I}^{2}$ statistic was used to describe the proportion of the variability that is due to heterogeneity rather than sampling error. Analyses were based upon intention-totreat (ITT) or completer analysis when ITT data was not available. When standard deviations of the outcomes were not given, they were calculated using standard errors and sample sizes. We were unable to assess publication bias by funnel plot due to the scant number of included studies. All statistical analyses were conducted using Review Manager 5.2 (Copenhagen: The Nordic Cochrane Centre, The Cochrane Collaboration, 2012). Apart from meta-analysis, the dose-response relationship was also plotted to determine the efficacy of tofacitinib at different doses at week 12. All studies were described as international multicenter trials except Tanaka et al.'s study [18] which was conducted solely in Japan. Therefore, sensitivity analysis was conducted by removing Tanaka et al.'s study [18] to examine the possible effects of differences in ethnicity.

\section{Results}

\section{Search results and study selection}

Figure 1 summarises the review flowchart in accordance with PRISMA statement [26]. The electronic search of EMBASE, the Cochrane Library and PubMed yielded a total of 283 studies. Four additional unpublished records were identified in the ClinicalTrials.gov, each with a different ClinicalTrial.gov identifier from the published reports (Additional file 2: Table S2). A total of 259 records were screened after the removal of the duplicates. After scanning the titles and abstracts, 216 records were removed as they were not clinical trials of tofacitinib. Full texts of 43 studies were retrieved for more detailed evaluation, of which 34 were then excluded since they were not related to the treatment of RA. Another study was excluded as it investigated pain, physical functioning and health status but not efficacy and safety measures of tofacitinib in the treatment of RA [31]. As a result, eight eligible studies were included in this systematic review, contributing a total sample size of 3,791. A standardised summary table of the included studies is presented in Table 1.

\section{Methodological quality}

All studies stated that they were double-blind, however half of the studies did not report the method of allocation sequence and concealment. Co-interventions and baseline characteristics were similar for the tofacitinib and placebo groups for all studies. All studies had potential risks of bias as some of the outcomes stated in the trial protocol were not reported. Another potential bias might be introduced by switching from placebo to active medication in some patients previously receiving placebo in four studies. This potential bias was addressed in two studies using the method of imputation of no response, with "advancement penalty" [22,23].

\section{Efficacy}

The doses used and the treatment duration are shown in Figures 2 and 3. ACR20 response rates were found to be significantly higher in those patients receiving tofacitinib versus placebo at doses $\geq 3 \mathrm{mg}$ bid. A non-significant dose-dependent response trend was observed from 1 to $5 \mathrm{mg}$ bid (Additional file 3: Figure S1). ACR20 response rates were significantly greater in tofacitinib treatment versus placebo at $5 \mathrm{mg}$ bid (RR 2.20; 95\% CI 1.58, 3.07) and $10 \mathrm{mg}$ bid (RR 2.38; 95\% CI 1.81, 3.14) after 12 weeks of treatment. The higher ACR20 response rates in patients receiving tofacitinib sustained at week 24 for $5 \mathrm{mg}$ bid (RR 1.94; 95\% CI 1.55, 2.44) and $10 \mathrm{mg}$ bid (RR 2.20; 95\% CI 1.76, 2.75) (Figure 2). Moreover, tofacitinib was also significantly more efficacious than placebo as measured by ACR50 response rate (Figure 3). ACR50 response rates were significantly greater in tofacitinib treatment at $5 \mathrm{mg}$ bid (RR 2.91; 95\% CI 2.03, 4.16) and $10 \mathrm{mg}$ bid (RR 3.32; 95\% CI 2.33, 4.72) after 12 weeks.

For the efficacy measures which were only reported in respective single studies, significantly higher ACR20 and ACR50 response rates were observed in patients receiving doses $\geq 5 \mathrm{mg}$ tofacitinib versus placebo at week 6,12 and 24 (Additional file 4: Figure S2). A significantly higher response rate was also observed in ACR50 for $3 \mathrm{mg}$ tofacitinib versus placebo at week 24 .

Fleischmann et al. [21] and van Vollenhoven et al. [22] also compared the efficacy of tofacitinib with adalimumab at month 3 and 6 respectively (Table 2). At month 3 , there was a statistically significant difference in ACR20 response rate in patients receiving $\geq 5 \mathrm{mg}$ bid tofacitinib versus adalimumab. At the dose of $5 \mathrm{mg}$ bid, the RR of ACR20 and ACR50 response rates were 1.65 $(95 \%$ CI 1.08, 2.53) and 1.95 (95\% CI 1.00, 3.80) in patients receiving tofacitinib versus adalimumab respectively. The corresponding figures at $10 \mathrm{mg}$ bid were 1.97 (95\% CI 1.32, 2.92) and 2.35 (95\% CI 1.26, 4.38) respectively. At month 6 , there were no significant differences in ACR20 response rates in patients receiving tofacitinib versus adalimumab.

\section{Safety}

The most commonly reported infections and immunerelated AEs during the 12-week tofacitinib treatment period are shown in Table 3 . There were no statistically significant differences in patients receiving tofacitinib versus placebo in the incidences of infections, neutropenia and withdrawal due to AEs. However, significantly 
Table 1 Characteristics of randomised controlled studies included in this meta-analysis

\begin{tabular}{|c|c|c|c|c|c|c|c|c|c|c|}
\hline Article & Region & $\begin{array}{l}\text { Study } \\
\text { duration, } \\
\text { weeks }\end{array}$ & Possible concomitant medication & $\begin{array}{l}\text { Dose of } \\
\text { tofacitinib }\end{array}$ & $\begin{array}{l}\text { No of patients } \\
\text { randomised }^{\mathrm{a}}\end{array}$ & $\begin{array}{l}\text { Mean } \\
\text { DAS28-3 } \\
\left(_{(C R P)}\right)^{a}\end{array}$ & $\begin{array}{l}\text { Mean } \\
\text { DAS28-4 } \\
\left(^{(E S R)^{a}}\right.\end{array}$ & $\begin{array}{l}\text { Mean HAQ- } \\
\mathrm{DI}^{\mathrm{a}}\end{array}$ & $\begin{array}{l}\text { Mean no of } \\
\text { swollen } \\
\text { joints }^{a}\end{array}$ & $\begin{array}{l}\text { Mean no of } \\
\text { tender joints }\end{array}$ \\
\hline \multirow{5}{*}{$\begin{array}{l}\text { Fleischmann } \\
\text { 2012a [21] }\end{array}$} & \multirow{5}{*}{$\begin{array}{l}\text { United States; } \\
\text { Europe; Latin } \\
\text { American; the } \\
\text { Republic of } \\
\text { Korea }\end{array}$} & \multirow[t]{5}{*}{24} & \multirow{5}{*}{$\begin{array}{l}\text { NSAIDs; antimalarial agents; opioids; } \\
\text { acetaminophen; oral glucocorticoids }\end{array}$} & \multirow{5}{*}{$\begin{array}{l}1,3,5,10 \\
15 \mathrm{mg} \mathrm{bid}\end{array}$} & Total: 386 & Placebo:5.6 & Placebo:6.6 & Placebo:1.54 & Placebo:16.9 & Placebo:25.9 \\
\hline & & & & & Placebo: 59 & & & & & \\
\hline & & & & & $\begin{array}{l}\text { tofacitinib: } 54 \\
51,49,61,57\end{array}$ & $\begin{array}{l}\text { tofacinitib: } \\
5.5,5.4,5.6 \\
5.5,5.5\end{array}$ & $\begin{array}{l}\text { tofacinitib: } \\
6.5,6.4,6.6 \\
6.5,6.5\end{array}$ & $\begin{array}{l}\text { tofacinitib: } \\
1.57,1.53 \\
1.40,1.49 \\
1.62\end{array}$ & $\begin{array}{l}\text { tofacinitib: } \\
\text { 16.7, 15.9, } \\
17.4,16.3 \\
16.9\end{array}$ & $\begin{array}{l}\text { tofacinitib: 27.0, } \\
24.6,27.1,25.7, \\
25.9\end{array}$ \\
\hline & & & & & $\begin{array}{l}\text { adalimumab } \\
40 \text { mg qow: } 53\end{array}$ & $\begin{array}{l}\text { adalimumab } \\
40 \text { mg qow: } \\
5.4\end{array}$ & $\begin{array}{l}\text { adalimumab } \\
40 \text { mg qow: } \\
6.3\end{array}$ & $\begin{array}{l}\text { adalimumab } \\
40 \text { mg qow: } \\
1.44\end{array}$ & $\begin{array}{l}\text { adalimumab } \\
40 \text { mg qow: } \\
14.9\end{array}$ & $\begin{array}{l}\text { adalimumab } \\
40 \text { mg qow: } \\
24.1\end{array}$ \\
\hline & & & & & $\begin{array}{l}\text { Randomised } \\
\text { but not } \\
\text { treated: } 2\end{array}$ & & & & & \\
\hline \multirow{4}{*}{$\begin{array}{l}\text { Fleischmann } \\
2012 b[25]\end{array}$} & \multirow{4}{*}{$\begin{array}{l}\text { United States; } \\
\text { Europe; Latin } \\
\text { America; Asia }\end{array}$} & \multirow[t]{4}{*}{24} & \multirow[t]{4}{*}{ NSAIDs; glucocorticoids; } & \multirow{4}{*}{$\begin{array}{l}5,10 \mathrm{mg} \\
\text { bid }\end{array}$} & Total: 611 & Placebo: 5.56 & Placebo: 6.65 & Placebo: 1.53 & Placebo: 17.3 & Placebo: 28.9 \\
\hline & & & & & $\begin{array}{l}\text { Placebob: 61, } \\
61\end{array}$ & $\begin{array}{l}\text { tofacitinib: } \\
5.68,5.60\end{array}$ & $\begin{array}{l}\text { tofacitinib: } \\
6.71,6.70\end{array}$ & $\begin{array}{l}\text { tofacitinib: } \\
1.53,1.50\end{array}$ & $\begin{array}{l}\text { tofacitinib: } \\
16.3,17.0\end{array}$ & $\begin{array}{l}\text { tofacitinib: } 29.4, \\
29.1\end{array}$ \\
\hline & & & & & $\begin{array}{l}\text { tofacitinib:243, } \\
245\end{array}$ & & & & & \\
\hline & & & & & $\begin{array}{l}\text { Randomised } \\
\text { but not } \\
\text { treated: } 1\end{array}$ & & & & & \\
\hline \multirow[t]{3}{*}{ Kremer 2009 [20] } & \multirow{3}{*}{$\begin{array}{l}\text { United States; } \\
\text { Canada; } \\
\text { Europe; Latin } \\
\text { American }\end{array}$} & \multirow{3}{*}{$\begin{array}{l}6 \\
\text { (treatment) } \\
+6 \text { (follow- } \\
\text { up) }\end{array}$} & \multirow{3}{*}{$\begin{array}{l}\text { NSAIDs; selective COX-2 inhibitors; } \\
\text { opioids; acetaminophen; oral } \\
\text { glucocorticoids }\end{array}$} & \multirow[t]{3}{*}{$\begin{array}{l}5,15 \\
30 \mathrm{mg} \text { bid }\end{array}$} & Total: 264 & Placebo: 6.0 & NA & Placebo: 1.7 & $\begin{array}{l}\text { Placebo: } \\
20.01\end{array}$ & Placebo: 30.3 \\
\hline & & & & & Placebo: 65 & & & & & \\
\hline & & & & & $\begin{array}{l}\text { tofacitinib: } 61 \\
69,69\end{array}$ & $\begin{array}{l}\text { tofacitinib: } \\
6.2,5.7,5.9\end{array}$ & & $\begin{array}{l}\text { tofacitinib: } \\
1.7,1.6,1.6\end{array}$ & $\begin{array}{l}\text { tofacitinib: } \\
21.1,16.2 \\
19.5\end{array}$ & $\begin{array}{l}\text { tofacitinib: } 32.3, \\
26.7,29.3\end{array}$ \\
\hline \multirow[t]{4}{*}{ Kremer 2012 [19] } & \multirow{4}{*}{$\begin{array}{l}\text { United States; } \\
\text { Europe; Latin } \\
\text { America }\end{array}$} & \multirow[t]{4}{*}{24} & \multirow[t]{4}{*}{ MTX (compulsory) } & \multirow{4}{*}{$\begin{array}{l}1,3,5,10 \\
15 \mathrm{mg} \text { bid, } \\
20 \mathrm{mg} \text { qd }\end{array}$} & Total: 509 & Placebo: 5.3 & Placebo: 6.1 & Placebo: 1.20 & Placebo: 15.7 & Placebo: 21.6 \\
\hline & & & & & Placebo: 69 & $\begin{array}{l}\text { tofacitinib: } \\
5.5,5.3,5.1 \\
5.3,5.4\end{array}$ & $\begin{array}{l}\text { tofacitinib: } \\
6.4,6.1,6.1 \\
6.4,6.2\end{array}$ & $\begin{array}{l}\text { tofacitinib: } \\
1.58,1.36 \\
1.44,1.33 \\
1.41\end{array}$ & $\begin{array}{l}\text { tofacitinib: } \\
6.5,15.7 \\
14.1,14.7 \\
15.3\end{array}$ & $\begin{array}{l}\text { tofacitinib:23.6, } \\
22.8,21.5,24.8, \\
23.7\end{array}$ \\
\hline & & & & & $\begin{array}{l}\text { tofacitinib: } 70 \text {, } \\
68,71,74,75\end{array}$ & $\begin{array}{l}20 \mathrm{mg} \text { qd: } \\
5.3\end{array}$ & $\begin{array}{l}20 \mathrm{mg} \text { qd: } \\
6.3\end{array}$ & $\begin{array}{l}20 \mathrm{mg} \text { qd: } \\
1.46\end{array}$ & $\begin{array}{l}20 \mathrm{mg} \text { qd: } \\
15.2\end{array}$ & 0 mg qd: 23.1 \\
\hline & & & & & $\begin{array}{l}20 \text { mg qd: } \\
80 \text { Randomised } \\
\text { but not } \\
\text { treated: } 2\end{array}$ & & & & & \\
\hline
\end{tabular}


Table 1 Characteristics of randomised controlled studies included in this meta-analysis (Continued)

\begin{tabular}{|c|c|c|c|c|c|c|c|c|c|c|}
\hline \multirow[t]{4}{*}{ Tanaka 2011 [18] } & \multirow[t]{4}{*}{ Japan } & \multirow[t]{4}{*}{12} & \multirow{3}{*}{$\begin{array}{l}\text { MTX supplemented with folic acid } \\
\text { (compulsory); }\end{array}$} & \multirow{3}{*}{$\begin{array}{l}1,3,5 \\
10 \mathrm{mg} \text { bid }\end{array}$} & Total: 140 & Placebo: 4.9 & Placebo: 5.9 & Placebo: 1.3 & Placebo: 13.8 & Placebo: 16.4 \\
\hline & & & & & Placebo: 28 & & & & & \\
\hline & & & & & $\begin{array}{l}\text { tofacitinib: } 28 \\
27,27,26\end{array}$ & $\begin{array}{l}\text { tofacitinib: } \\
5.0,5.1,5.0 \\
4.9\end{array}$ & $\begin{array}{l}\text { tofacitinib: } \\
6.1,6.1,6.0 \\
5.9\end{array}$ & $\begin{array}{l}\text { tofacitinib: } \\
1.1,1.3,1.2 \\
1.2\end{array}$ & $\begin{array}{l}\text { tofacitinib: } \\
\text { 13.2, 15.1, } \\
15.6,15.1\end{array}$ & $\begin{array}{l}\text { tofacitinib: } 16.4, \\
16.2,17.8,15.4\end{array}$ \\
\hline & & & $\begin{array}{l}\text { NSAIDs;selective COX-2 inhibitors; } \\
\text { glucocorticoids }\end{array}$ & & $\begin{array}{l}\text { Randomised } \\
\text { but not } \\
\text { treated: } 4\end{array}$ & & & & & \\
\hline \multirow{4}{*}{$\begin{array}{l}\text { van } \\
\text { Vollenhoven2012 } \\
{[22]}\end{array}$} & \multirow{4}{*}{$\begin{array}{l}\text { North America; } \\
\text { Latin America; } \\
\text { Europe; etc. }\end{array}$} & \multirow[t]{4}{*}{52} & \multirow[t]{4}{*}{ MTX (compulsory) 5, $10 \mathrm{mg}$ bid } & & Total: 717 & $\begin{array}{l}\text { Placeboc: } 5.6, \\
5.3\end{array}$ & $\begin{array}{l}\text { Placeboc: } 6.6, \\
6.3\end{array}$ & $\begin{array}{l}\text { Placebo }{ }^{c}: 1.5, \\
1.4\end{array}$ & $\begin{array}{l}\text { Placeboc: } \\
16.9,16.4\end{array}$ & $\begin{array}{l}\text { Placeboc: } 26.6, \\
28.1\end{array}$ \\
\hline & & & & & $\begin{array}{l}\text { Placebo }: 56, \\
52\end{array}$ & $\begin{array}{l}\text { tofacitinib: } \\
5.4,5.4\end{array}$ & $\begin{array}{l}\text { tofacitinib: } \\
6.6,6.5\end{array}$ & $\begin{array}{l}\text { tofacitinib: } \\
1.5,1.5\end{array}$ & $\begin{array}{l}\text { tofacitinib: } \\
16.7,15.8\end{array}$ & $\begin{array}{l}\text { tofacitinib: } 28.5, \\
26.1\end{array}$ \\
\hline & & & & & $\begin{array}{l}\text { tofacitinib: 204, } \\
201\end{array}$ & $\begin{array}{l}\text { adalimumab } \\
40 \text { mg qow: } \\
5.3\end{array}$ & $\begin{array}{l}\text { adalimumab } \\
40 \text { mg qow: } \\
6.4\end{array}$ & $\begin{array}{l}\text { adalimumab } \\
40 \text { mg qow: } \\
1.5\end{array}$ & $\begin{array}{l}\text { adalimumab } \\
40 \mathrm{mg} \\
\text { qow: } 16.4\end{array}$ & $\begin{array}{l}\text { adalimumab } \\
40 \text { mg qow: } \\
26.7\end{array}$ \\
\hline & & & & & $\begin{array}{l}\text { adalimumab } \\
40 \text { mg qow: } \\
204\end{array}$ & & & & & \\
\hline \multirow{3}{*}{$\begin{array}{l}\text { Burmester } 2013 \\
{[24]}\end{array}$} & \multirow{3}{*}{$\begin{array}{l}\text { North America, } \\
\text { Europe, Latin } \\
\text { America, etc. }\end{array}$} & \multirow[t]{3}{*}{24} & \multirow{3}{*}{$\begin{array}{l}\text { MTX (compulsory); antimalarial therapy; } \\
\text { No other DMARDs (non-biological or } \\
\text { biological) were permitted NSAIDs, } \\
\text { selective cyclooxygenase-2 inhibitors, or } \\
\text { glucocorticoids }\end{array}$} & \multirow{3}{*}{$\begin{array}{l}5,10 \mathrm{mg} \\
\text { bid }\end{array}$} & Total: 399 & Placebo: 5.4 & Placebo: 6.4 & Placebo: 1.6 & Placebo: 17.2 & Placebo: 28.2 \\
\hline & & & & & $\begin{array}{l}\text { Placebo } o^{b}: 66, \\
66\end{array}$ & $\begin{array}{l}\text { tofacitinib: } \\
5.4,5.3\end{array}$ & $\begin{array}{l}\text { tofacitinib: } \\
6.5,6.4\end{array}$ & $\begin{array}{l}\text { tofacitinib: } \\
1.6,1.5\end{array}$ & $\begin{array}{l}\text { tofacitinib: } \\
\text { 16.2, } 16.6\end{array}$ & $\begin{array}{l}\text { tofacitinib: } 28.4, \\
27.6\end{array}$ \\
\hline & & & & & $\begin{array}{l}\text { tofacitinib: } 133 \\
134\end{array}$ & & & & & \\
\hline \multirow[t]{3}{*}{$\begin{array}{l}\text { Van der Heijde } \\
2013[23]\end{array}$} & \multirow{3}{*}{$\begin{array}{l}\text { America, } \\
\text { Europe, Asia, } \\
\text { Australia }\end{array}$} & \multirow[t]{3}{*}{104} & \multirow[t]{3}{*}{ MTX (compulsory); NSAIDs; corticosteroids } & \multirow[t]{3}{*}{$\begin{array}{l}5,10 \mathrm{mg} \\
\text { bid }\end{array}$} & Total: 797 & $\begin{array}{l}\text { Placeboc: } \\
5.14,5.18\end{array}$ & $\begin{array}{l}\text { Placeboc: } \\
6.25,6.29\end{array}$ & $\begin{array}{l}\text { Placebo }{ }^{c}: 1.4 \\
1.23\end{array}$ & $\begin{array}{l}\text { Placeboc: } \\
14.0,14.5\end{array}$ & $\begin{array}{l}\text { Placeboc: } 23.3, \\
22.6\end{array}$ \\
\hline & & & & & $\begin{array}{l}\text { Placeboc: } 81 \\
79\end{array}$ & $\begin{array}{l}\text { tofacitinib: } \\
5.22,5.20\end{array}$ & $\begin{array}{l}\text { tofacitinib: } \\
6.34,6.25\end{array}$ & $\begin{array}{l}\text { tofacitinib: } \\
1.41,1.39\end{array}$ & $\begin{array}{l}\text { tofacitinib: } \\
14.1,14.4\end{array}$ & $\begin{array}{l}\text { tofacitinib: } 24.1, \\
23.0\end{array}$ \\
\hline & & & & & $\begin{array}{l}\text { tofacitinib: } 321 \\
316\end{array}$ & & & & & \\
\hline
\end{tabular}


fewer patients withdrew from tofacitinib than placebo (RR 0.60; 95\% CI 0.45, 0.78). Similarly, the patient withdrawal rate due to lack of efficacy was significantly lower in the patients receiving tofacitinib versus placebo ( $R R$ 0.18; 95\% CI 0.09, 0.35).

The mean neutrophil count significantly declined in patients receiving tofacitinib versus placebo. The mean serum creatinine was found to be significantly higher for tofacitinib $10 \mathrm{mg}$ bid versus placebo. The mean percentage change of HDL/LDL was significant higher in patients receiving tofacitinib versus placebo. The RRs of the mean changes of ALT $>1$ ULN and AST $>1$ ULN were statistically significant (Additional file 5: Table S3).

\section{Sensitivity analysis}

The RR of ACR20 response rate did not significantly change with the exclusion of data from Tanaka et al. [18]. For the tofacitinib treatment at $3 \mathrm{mg}$ bid, the RR slightly reduced from 2.20 (95\% CI $1.20,4.04)$ to 1.65 (95\% CI 1.18, 2.30), however, the heterogeneity was significantly reduced to $0 \%$ (Additional file 6: Table S4). Similarly, the RR of ACR20 response rate for the tofacitinib treatment at $5 \mathrm{mg}$ bid $(2.20 ; 95 \% \mathrm{CI} 1.58$, 3.07) did not change significantly $(1.94 ; 95 \%$ CI 1.55 , 2.43 ) but led to a reduction in heterogeneity. Although the inclusion of Tanaka et al. [18] led to substantial heterogeneity, it did not materially alter the conclusion. No 


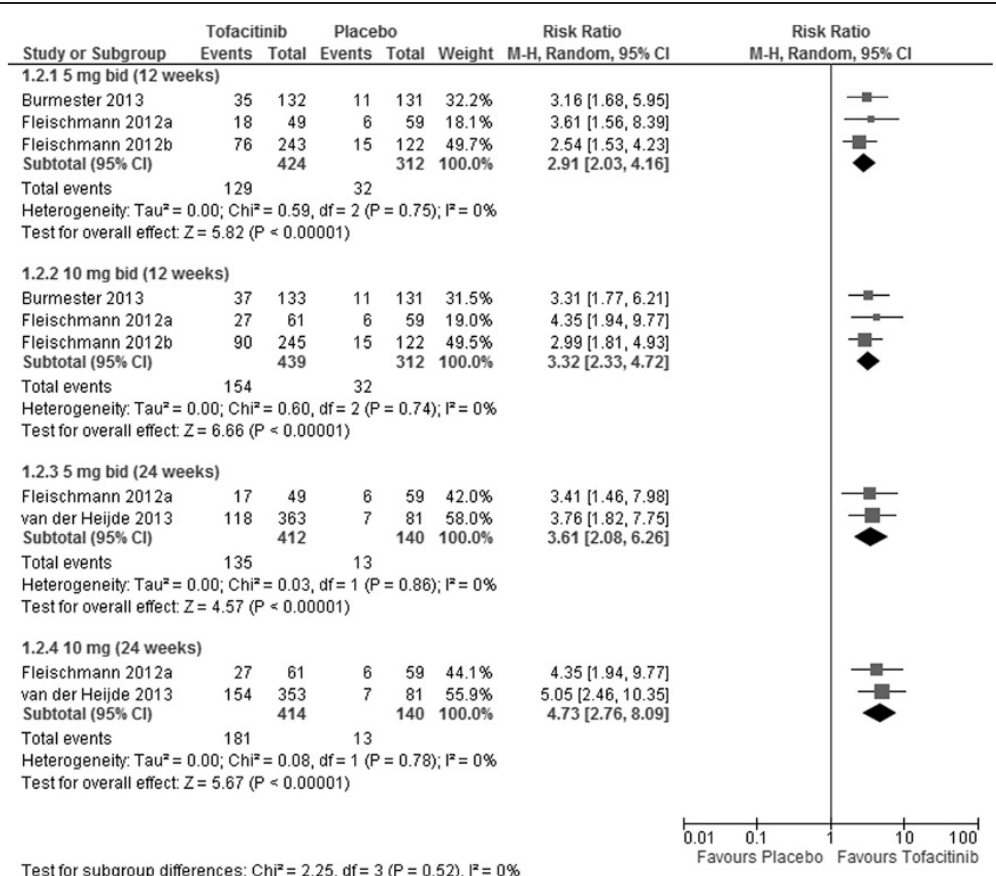

Test for subaroup differences: $\mathrm{Chi}^{2}=2.25, \mathrm{df}=3(\mathrm{P}=0.52) \cdot \mathrm{I}^{2}=0 \%$

Figure 3 ACR50 response rates for $5 \mathrm{mg}$ bid and $10 \mathrm{mg}$ bid of tofacitinib at week 12 and week 24 .

data for ACR50 response rate was available for sensitivity analysis.

\section{Discussion}

We undertook a rigorous systematic review and metaanalysis using independent reviewers for data extraction and statistical analysis. To our knowledge, this is the first systematic review of the RCTs investigating tofacitinib in the treatment of RA and included all relevant published RCTs to date. The meta-analysis of the clinical trials of tofacitinib in adult patients with RA showed that the use of tofacitinib $5 \mathrm{mg}$ bid resulted in statistically significant higher ACR20 and ACR50 response rates compared with placebo. A non-statistically significant dose-dependent effect was observed in tofacitinib treatment at 1,3,5 mg bid while the effect appeared to be saturated when the dose reached $5 \mathrm{mg}$ bid. In consistent with our result, the FDA has approved the clinical dose of $5 \mathrm{mg}$ bid for the treatment of adults with moderately to severely active RA [32].

Apart from the comparison of efficacy in different doses, our study also compared the efficacy of tofacitinib with one of the biologics, adalimumab. However, similar to many newly marketed drugs, most of the published studies were placebo-controlled trials. Two clinical trials comparing the efficacy of tofacitinib with adalimumab

Table 2 Risk ratios of ACR20/50 response rates of tofacitinib vs.40 mg adalimumab at month 3 [21] and 6 [22]

\begin{tabular}{|c|c|c|c|c|}
\hline \multirow[b]{2}{*}{ Dose of tofacitinib } & \multicolumn{2}{|l|}{ ACR20 } & \multicolumn{2}{|l|}{ ACR50 } \\
\hline & Sample size (tofacitinib, adalimumab) & Risk ratio $[95 \% \mathrm{Cl}]$ & Sample size (tofacitinib, adalimumab) & Risk ratio $[95 \% \mathrm{Cl}]$ \\
\hline \multicolumn{5}{|l|}{ Month 3} \\
\hline $1 \mathrm{mg}$ bid & 54,53 & $0.88[0.52,1.50]$ & 54,53 & $0.59[0.23,1.51]$ \\
\hline $3 \mathrm{mg}$ bid & 51,53 & $1.09[0.67,1.80]$ & 51,53 & $1.25[0.59,2.63]$ \\
\hline $5 \mathrm{mg} \mathrm{bid}$ & 49,53 & $1.65[1.08,2.53]$ & 49,53 & $1.95[1.00,3.80]$ \\
\hline $10 \mathrm{mg}$ bid & 61,53 & $1.97[1.32,2.92]$ & 61,53 & $2.35[1.26,4.38]$ \\
\hline $15 \mathrm{mg}$ bid & 57,53 & $2.01[1.35,2.98]$ & 57,53 & $2.70[1.46,4.98]$ \\
\hline \multicolumn{5}{|l|}{ Month 6, } \\
\hline $5 \mathrm{mg} \mathrm{bid}$ & 196, 199 & $1.09[0.89,1.33]$ & N/A & N/A \\
\hline $10 \mathrm{mg}$ bid & 196: 199 & $1.11[0.91,1.36]$ & N/A & $\mathrm{N} / \mathrm{A}$ \\
\hline
\end{tabular}

bid twice daily; Cl confidence interval; N/A, not applicable. 
Table 3 Adverse events with tofacitinib at week 12 and withdrawal from trials

\begin{tabular}{|c|c|c|c|c|c|c|}
\hline \multirow[b]{2}{*}{ Adverse events } & \multicolumn{3}{|c|}{$5 \mathrm{mg}$ bid } & \multicolumn{3}{|c|}{$10 \mathrm{mg}$ bid } \\
\hline & $\begin{array}{l}\text { No. of } \\
\text { studies }\end{array}$ & $\begin{array}{c}\text { Sample size } \\
\text { (tofacitinib, placebo) }\end{array}$ & $\begin{array}{l}\text { Risk ratio } \\
{[95 \% \mathrm{Cl}]}\end{array}$ & $\begin{array}{l}\text { No. of } \\
\text { studies }\end{array}$ & $\begin{array}{c}\text { Sample size } \\
\text { (tofacitinib, placebo) }\end{array}$ & $\begin{array}{l}\text { Risk ratio } \\
{[95 \% \mathrm{Cl}]}\end{array}$ \\
\hline \multicolumn{7}{|l|}{ Infection } \\
\hline $\begin{array}{l}\text { Upper respiratory tract } \\
\text { infection }\end{array}$ & 4 & 901,522 & $1.12[0.61,2.05]$ & 4 & 896,522 & $0.77[0.40,1.49]$ \\
\hline Urinary tract infection & 4 & 901,522 & $1.22[0.58,2.57]$ & 4 & 901,522 & $1.01[0.45,2.27]$ \\
\hline Bronchitis & 4 & 901,522 & $0.80[0.25,2.56]$ & 4 & 896,522 & $1.01[0.34,2.96]$ \\
\hline Nasopharyngitis & 5 & 928,550 & $1.57[0.51,4.83]$ & 5 & 922,550 & $1.67[0.82,3.39]$ \\
\hline Influenza & 3 & 768,390 & $0.37[0.08,1.64]$ & 3 & 762,390 & $1.25[0.25,6.20]$ \\
\hline Pharyngitis & 2 & 231,136 & $0.01[-0.01,0.03]$ & 2 & 227,136 & $0.03[-0.09,0.14]$ \\
\hline \multicolumn{7}{|l|}{ Immune system } \\
\hline Neutropenia $^{a}$ & 4 & 826,482 & $1.41[0.55,3.61]$ & 4 & 830,482 & $1.73[0.68,4.38]$ \\
\hline Rash & 3 & 552,296 & $0.32[0.04,2.61]$ & 3 & 543,296 & $2.51[0.63,9.93]$ \\
\hline Withdrawal & No. of studies & Tofacitinib N (\%) & Placebo N (\%) & & & Risk ratio $[95 \% \mathrm{Cl}]$ \\
\hline All causes & 5 & $158(12.31 \%)$ & $59(16.71 \%)$ & & & $0.60[0.45,0.78]$ \\
\hline Adverse events & 5 & $50(3.89 \%)$ & $8(2.27 \%)$ & & & $1.43[0.68,3.03]$ \\
\hline Lack of efficacy & 5 & $16(1.25 \%)$ & $20(5.67 \%)$ & & & $0.18[0.09,0.35]$ \\
\hline
\end{tabular}

bid twice daily; Cl confidence interval; ${ }^{a}$ mild, $1500-1999$ cells $/ \mathrm{mm}^{3}$.

were identified. Our findings showed that ACR20 and ACR50 response rates were statistically significant higher in patients receiving tofacitinib versus adalimumab at month 3, except the ACR50 response rate of tofacitinib $5 \mathrm{mg}$ bid treatment. However, the ACR20 response rate of tofacitinib was similar to that of adalimumab in a period of 6 months. Aaltonen et al. [33] recently conducted a meta-analysis to compare the response rates (ACR20 and ACR50) of TNF inhibitors with placebo group. They reported the RR of ACR20 response rate at month 3 were 2.24 (95\% CI 1.63, 3.08) and 2.50 (95\% CI $1.90,3.30)$ at month 6 . Asltonen et al.'s results are also comparable to our results on tofacitinib $5 \mathrm{mg}$ bid treatment at month 3 (RR 2.44; 95\% CI 1.58, 3.77) and at month 6 (RR 1.87; 95\% CI 1.42, 2.48). Similarly, RR of ACR50 response rate at month 3 reported by Aaltonen et al. was 4.16 (95\% CI 2.44, 7.09) which is also comparable to ours in tofacitinib (5 or $10 \mathrm{mg}$ bid) at month 3 (RR 3.05; 95\% CI 2.25, 4.14). The current available evidence seemed to support the efficacy of tofacitinib in the short-term treatment of RA, which may be comparable to TNF inhibitors. However, further head-to-head direct comparison studies are needed to confirm the results.

Unlike the biologics which are administered by injection, tofacitinib is a small molecule which can be administered orally. Although tofacitinib is not currently licensed for children, an oral treatment is likely to be well received by children with MTX-resistant RA. In accordance with the requirements of the new European and FDA paediatric regulations, the manufacturer should plan on conducting paediatric clinical trials so that data will be available in the future to guide the use of tofacitinib in children.

In our meta-analysis, the results showed no statistically significant difference in the outcome of AEs in the tofacitinib group versus placebo but some laboratory abnormalities were observed in short-term studies. We found significantly higher mean serum creatinine in the tofacitinib group and it was also in line with a review reporting higher incidence rate of blood creatinine elevation in tofacitinib treatment group compared to comparator group [34]. However, this did not result in patient withdrawal at week 12 shown in our meta-analysis. Similarly, there was a significantly higher risk of ALT/AST $>1$ ULN in the tofacitinib group versus placebo. One study reported that four patients discontinued in tofacitinib treatment group but none in the placebo group due to increases of AST and ALT levels [18]. Moreover, tofacitinib has the potential to cause immunosuppression, inducing serious infections and malignancies. This possibility is supported by its pharmacological action which acts as a JAK1/3 inhibitor. A conference abstract showed a statistically significant higher risk of infection due to the decrease in absolute lymphocyte counts [35]. Significantly lower neutrophil counts were also found in tofacitinib treatment versus placebo, but no patients' discontinuation was reported. The most common infection-related AEs reported in tofacitinib treatment were upper respiratory tract infections, urinary tract infections and nasopharyngitis [27]. Tuberculosis was also reported in four patients receiving tofacitinib $10 \mathrm{mg}$ bid treatment $[22,23,25]$. With respect to malignancies, lung cancer $[22,23,25]$ and renal cell carcinoma [21] were descriptively 
reported in the included studies. Another conference abstract summarising RCTs and long-term extension studies reported higher incidence rate when the duration of exposure to tofacitinib is longer. It also reported statistically significant higher risk of lung cancer in the tofacitinib group compared with the Surveillance Epidemiology and End Result database covering the general population [36]. However more studies are needed to confirm the above findings and also investigate the risk of other malignancy associated with long-term tofacitinib treatment. Apart from malignancies, the European Medicines Agency also suggested that cardiovascular problems (largely related to adverse lipid profile) should be specifically monitored in patients with RA in clinical trials [8]. A meta-analysis reported statistically significant higher risk of hypercholesterolaemia (RR $1.70 ; 95 \% \mathrm{CI} 1.10,2.63)$ in tofacitinib treatment group when compared to the comparator group [34]. A significant increase in LDL/HDL was also noted in our meta-analysis. Of note, in one study [24], the results for changes in LDL/ HDL reported in the main text was not consistent with the supplementary figures, hence was excluded from the analysis. Cardiovascular outcomes such as congestive cardiac failure, chest pain and chest discomfort were also descriptively reported in the included studies $[19,21]$.

Despite the laboratory abnormalities, the clinical significance was still unclear as too few patients discontinued and no statistically significant difference was observed in all RRs of AEs. However, long-term pharmacovigilance studies are needed to explore the clinical significance on the laboratory abnormalities and confirm the long-term safety.

Our results showed that more patients withdrew from the placebo group than from the tofacitinib group (Table 3). The above results can be explained by the fact that significantly more patients in the placebo group withdrew due to lack of efficacy. Although there was a higher withdrawal rate due to AEs in the tofacitinib group than that of placebo group, the difference was not statistically significant. The above results further support that tofacitinib has a favorable risk/benefit ratio for short-term use.

There are several potential limitations in our metaanalysis. Firstly, all published studies have reported statistically significant higher ACR20 and ACR50 response rates in patients receiving tofacitinib when compared to placebo and all these clinical trials were sponsored by the manufacturer. At the time of the analysis, we also identified four more completed registered trials from the ClinicalTrials.gov since 2010 but peer-reviewed published results were yet to be available (Additional file 2: Table S2). Consequently, the possibility of publication bias and time-lag bias cannot be excluded. Although we have generated funnel plots to access publication bias, the paucity of the literature makes it difficult to warrant its reliability. Secondly, the patient in the included studies all had an inadequate response to
MTX treatment, which may limit the generalisability of study findings to DMARD naive patients. Thirdly, there is substantial statistical heterogeneity between studies in the outcomes of ACR20 and ACR50 response rates. This apparent heterogeneity was likely to be attributed to data from a single study by the Tanaka et al. [18]. However, sensitivity analysis showed that its exclusion resulted in reduction in heterogeneity without materially affecting the overall conclusions. The setting of this study was similar to all other studies in terms of concomitant medication and study duration. However we observed a high RR of ACR20 response rate, which implied that the heterogeneity may be due to the difference in study populations as this study was conducted among the Japanese population only. The other studies were conducted internationally, mainly in North America. Pharmacogenomics studies are recommended to investigate the apparent differences in efficacy.

In addition, some important information was not reported in the included studies, which limit our further understanding of the efficacy and safety of tofacitinib treatment in some circumstances. First, data according to different age groups should be reported. The manufacturer reported that elderly people ( $\geq 65$ years) receiving tofacitinib might have a higher risk of developing serious infections and more severe RA symptoms, which may render different efficacy and safety of tofacitinib [27]. However, there was a lack of published information reporting the outcomes of this specific age group. Second, radiographic outcomes such as erosions, joint space narrowing and Sharp van der Heijde should be reported at least at baseline, during and at the end of the trial for assessing the efficacy [10] but they were not reported in the included trials.

\section{Conclusions}

In conclusion, tofacitinib is more effective than placebo in the treatment of MTA-resistant RA up to 24 weeks. Tofacitinib is well tolerated as no statistically significant AEs impacting the immune or hematologic system were observed in short-term studies compared with placebo. Despite significantly lower neutrophil counts in tofacitinib group, there were no associated treatment withdrawals. However, further studies on long-term efficacy and pharmacovigilance studies are still needed to support long-term use.

\section{Additional files}

Additional file 1: Table S1. Assessment of risk of bias in accordance to Cochrane Collaboration's tool.

Additional file 2: Table S2. Completed clinical trials identified from the ClinicalTrials.gov (not included in this systematic review as results were not available).

Additional file 3: Figure S1. Risk ratios of ACR20 response rates of tofacitinib versus placebo at week 12 . 
Additional file 4: Figure S2. ACR20 and ACR 50 response rates reported in three independent studies* ${ }^{*}$ The three independent studies were Kremer 2009 [20], Kremer 2012 [19] and Fleischmann 2012a [21].

Additional file 5: Table S3. Laboratory findings with tofacitinib treatment at week 12 .

Additional file 6: Table S4. Sensitivity analysis (exclusion of Tanaka et al [18]) of risk ratios of ACR20 response rates.

\section{Abbreviations}

ACR: American College of Rheumatology Scale; AE: Adverse event; AST: Aspartate aminotransferase; ALT: Alanine aminotransferase; BID: Twice daily; Cl: Confidence interval; DAS: Disease activity score; DMARD: Diseasemodifying antirheumatic drug; FDA: US food and drug administration; HAQDI: Health assessment questionnaire disability index; HDL: High-density lipoprotein; ICTRP: International clinical trials registry platform; ITT: Intentionto-treat; JAK: Janus Kinase; LDL: Low-density lipoprotein; MTX: Methotrexate; NICE: National institute for health and clinical excellence; NSAIDs: Nonsteroidal anti-inflammatory drugs; PRISMA: Preferred reporting items for systematic reviews and meta-analyses; RA: Rheumatoid arthritis; RCT: Randomised controlled trials; RR: Relative risk; TNF: Tumor necrosis factor; ULN: Upper limit of normal.

\section{Competing interests}

The authors declare that they have no competing interests.

\section{Authors' contributions}

ICKW, EWC, YH, AYSW and CSLC planned the systematic review and developed the methodology. CSLC, WCYL, KKCM, YH, AYSW, and AJW contributed to extract and interpreted the data. WCYL, KKCM, AYSW and AJW involved in the statistical analysis. YH, AYSW, WCYL, EWC and ICKW drafted the article. All authors revised the article critically for important intellectual content and approved the final manuscript.

Received: 11 May 2013 Accepted: 11 October 2013

Published: 18 October 2013

\section{References}

1. Majithia V, Geraci SA: Rheumatoid arthritis: diagnosis and management. Am J Med 2007, 120:936-939.

2. Nakamura T, Higashi S, Tomoda K, Tsukano M, lyama K: Cutaneous nodules in patients with rheumatoid arthritis: a case report and review of literatures. Clin Rheumatol 2011, 30:719-722.

3. Bongartz T, Nannini C, Medina-Velasquez Y, Achenbach S, Crowson C, Ryu J, Vassallo R, Gabriel S, Matteson E: Incidence and mortality of interstitial lung disease in rheumatoid arthritis: a population-based study. Arthritis Rheum 2010, 62:1583-1591.

4. Voskuyl A: The heart and cardiovascular manifestations in rheumatoid arthritis. Rheumatology 2006, 45:iv4-iv7.

5. Balint G, Balint P: Felty's Syndrome. Best Pract Res Clin Rheumatol 2004, 18:631-645

6. Rheumatoid arthritis: the management of rheumatoid arthritis in adults. NICE clinical guideline 79. http://www.nice.org.uk/nicemedia/pdf/ CG79NICEGuideline.pdf.

7. American College of Rheumatology Subcommittee on Rheumatoid Arthritis G: Guidelines for the management of rheumatoid arthritis: 2002 update. Arthritis Rheum 2002, 46:328-346.

8. Guideline on clinical investigation of medicinal products other than NSAIDs for treatment of rheumatoid arthritis. http://www.ema.europa.eu/docs/en_GB/ document_library/Scientific_guideline/2011/12/WC500119785.pdf.

9. Wolfe F, Mitchell DM, Sibley JT, Fries JF, Bloch DA, Williams CA, Spitz PW, Haga M, Kleinheksel SM, Cathey MA: The mortality of rheumatoid arthritis. Arthritis Rheum 1994, 37:481-494.

10. Singh JA, Furst DE, Bharat A, Curtis JR, Kavanaugh AF, Kremer JM, Moreland LW, O'Dell J, Winthrop KL, Beukelman T, et al: 2012 Update of the 2008 american college of rheumatology recommendations for the use of disease-modifying antirheumatic drugs and biologic agents in the treatment of rheumatoid arthritis. Arthritis Care Res 2012, 64:625-639.

11. Wilkie WS, Schwieterman P: Strategies for the management of rheumatoid arthritis. Orthopedics 2012, 35:125-130.
12. Krishnaswami S, Kudlacz E, Wang R, Chan G: A supratherapeutic dose of the janus kinase inhibitor tasocitinib (CP-690,550) does not prolong QTC interval in healthy participants. J Clin Pharmacol 2011, 51:1256-1263.

13. FDA approves xeljanz for rheumatoid arthritis. http://www.fda.gov/ NewsEvents/Newsroom/PressAnnouncements/ucm327152.htm.

14. Fox DA: Kinase inhibition-a new approach to the treatment of rheumatoid arthritis. N Engl J Med 2012, 367:565-567.

15. Kyttaris VC: Kinase inhibitors: a new class of antirheumatic drugs. Drug Des Devel Ther 2012, 6:245-250.

16. Yamaoka K, Kubo S, Maeshima K, Sonomoto K, Nakano K, Sawamukai N, Nawata M: Tofacitinib reduces interleukin-6 and matrix metalloproteinase-3 production and inhibits cartilage destruction in rheumatoid arthritis, Annual scientific meeting of the american college of rheumatology and association of rheumatology health professionals 2011; october. Chicago, IL United States: Arthritis Rheum; 2011. 10 SUPPL. 11.

17. Yarilina A, Xu K, Chan C, Ivashkiv LB: Regulation of inflammatory responses in tumor necrosis factor-activated and rheumatoid arthritis synovial macrophages by JAK inhibitors. Arthritis Rheum 2012, 64:3856-3866.

18. Tanaka Y, Suzuki M, Nakamura H, Toyoizumi S, Zwillich SH: Phase II study of tofacitinib (CP-690,550) combined with methotrexate in patients with rheumatoid arthritis and an inadequate response to methotrexate. Arthritis Care Res 2011, 63:1150-1158.

19. Kremer JM, Cohen S, Wilkinson BE, Connell CA, French JL, Gomez-Reino J, Gruben D, Kanik KS, Krishnaswami S, Pascual-Ramos V, et al: A phase IIb dose-ranging study of the oral JAK inhibitor tofacitinib (CP-690,550) versus placebo in combination with background methotrexate in patients with active rheumatoid arthritis and an inadequate response to methotrexate alone. Arthritis Rheum 2012, 64:970-981.

20. Kremer JM, Bloom BJ, Breedveld FC, Coombs JH, Fletcher MP, Gruben D, Krishnaswami S, Burgos-Vargas R, Wilkinson B, Zerbini CA, Zwillich SH: The safety and efficacy of a JAK inhibitor in patients with active rheumatoid arthritis: results of a double-blind, placebo-controlled phase lla trial of three dosage levels of CP-690,550 versus placebo. Arthritis Rheum 2009, 60:1895-1905.

21. Fleischmann R, Cutolo M, Genovese MC, Lee EB, Kanik KS, Sadis S, Connell CA, Gruben D, Krishnaswami S, Wallenstein G, et al: Phase Ilb dose-ranging study of the oral JAK inhibitor tofacitinib (CP-690,550) or adalimumab monotherapy versus placebo in patients with active rheumatoid arthritis with an inadequate response to disease-modifying antirheumatic drugs. Arthritis Rheum 2012, 64:617-629.

22. van Vollenhoven RF, Fleischmann R, Cohen S, Lee EB, Garcia Meijide JA, Wagner S, Forejtova S, Zwillich SH, Gruben D, Koncz T, et al: Tofacitinib or adalimumab versus placebo in rheumatoid arthritis. N Engl J Med 2012, 367:508-519.

23. van der Heijde D, Tanaka Y, Fleischmann R, Keystone E, Kremer J, Zerbini C Cardiel MH, Cohen S, Nash P, Song YW, et al: Tofacitinib (CP-690,550) in patients with rheumatoid arthritis on methotrexate: 12-month data from a 24-month phase 3 randomized radiographic study. Arthritis Rheum 2013, 65:559-570.

24. Burmester GR, Blanco R, Charles-Schoeman C, Wollenhaupt J, Zerbini C, Benda B, Gruben D, Wallenstein G, Krishnaswami S, Zwillich SH, et al: Tofacitinib (CP-690,550) in combination with methotrexate in patients with active rheumatoid arthritis with an inadequate response to tumour necrosis factor inhibitors: a randomised phase 3 trial. Lancet 2013, 381:9-15.

25. Fleischmann R, Kremer J, Cush J, Schulze-Koops H, Connell CA, Bradley JD, Gruben D, Wallenstein GV, Zwillich SH, Kanik KS: Placebo-controlled trial of tofacitinib monotherapy in rheumatoid arthritis. N Engl J Med 2012, 367:495-507.

26. Moher D, Liberati A, Tetzlaff J, Altman DG, Group P: Preferred reporting items for systematic reviews and meta-analyses: the PRISMA statement BMJ 2009, 339:332-336.

27. Tofacitinib for the treatment of rheumatoid arthritis. NDA 203214. Briefing document for the May 9, 2012, meeting of the arthritis advisory committee. http://www.fda.gov/downloads/AdvisoryCommittees/ CommitteesMeetingMaterials/Drugs/ArthritisAdvisoryCommittee/ UCM302960

28. Felson D, Anderson J, Boers M: American college of rheumatology. Preliminary definition of improvement in rheumatoid arthritis. Arthritis Rheum 1995, 38:727-735.

29. Chapter 8: assessing risk of bias in included studies. http://www.cochrane.org/ sites/default/files/uploads/handbook/Handbook510pdf_Ch08_RiskOfBias.pdf. 
30. Ades AE, Lu G, Higgins JPT: The interpretation of random-effects metaanalysis in decision models. Med Decis Making 2005, 25:646-654.

31. Coombs JH, Bloom BJ, Breedveld FC, Fletcher MP, Gruben D, Kremer JM, Burgos-Vargas R, Wilkinson B, Zerbini CA, Zwillich SH: Improved pain, physical functioning and health status in patients with rheumatoid arthritis treated with CP-690,550, an orally active janus kinase (JAK) inhibitor: results from a randomised, double-blind, placebo-controlled trial. Ann Rheum Dis 2010, 69:413-416.

32. Highlights of prescribing information, XELJANZ ${ }^{\circledast}$ (tofacitinib) tablets for oral administration initial U.S. Approval http://www.accessdata.fda.gov/ drugsatfda_docs/label/2012/203214s000lbl.pdf.

33. Aaltonen K, Virkki LM, Malmivaara A, Konttinen YT, Nordstrom DC, Blom M: Systematic review and meta-analysis of the efficacy and safety of existing TNF blocking agents in treatment of rheumatoid arthritis. PLoS One 2012, 7:e30275.

34. Salgado E, Maneiro JR, Carmona L, Gomez-Reino JJ: Safety profile of protein kinase inhibitors in rheumatoid arthritis: systematic review and meta-analysis. Ann Rheum Dis 2013. Apr 18. [Epub ahead of print].

35. van Vollenhoven R, Riese R, Krishnaswami S, Kawabata T, Fosser $C$, Rottinghaus S, Lamba M, Zwillich SH, Bradley J: Relationship between lymphocyte count and risk of infection in rheumatoid arthritis patients treated with tofacitinib. In Proceedings of the Annual Congress of the European League Against Rheumatism; Madrid, Spain; 2013.

36. Mariette X, Curtis JR, Lee EB, Riese R, Kaplan I, Chew R, Geier J, Wang L, Bradley JD: Tofacitinib (CP-690,550), an oral janus kinase inhibitor: analysis of malignancies across the rheumatoid arthritis clinical programme- poster tours. In Proceedings of the Annual Congress of the European League Against Rheumatism; Berlin, Germany; 2012.

doi:10.1186/1471-2474-14-298

Cite this article as: He et al:: Efficacy and safety of tofacitinib in the treatment of rheumatoid arthritis: a systematic review and metaanalysis. BMC Musculoskeletal Disorders 2013 14:298.

\section{Submit your next manuscript to BioMed Central and take full advantage of:}

- Convenient online submission

- Thorough peer review

- No space constraints or color figure charges

- Immediate publication on acceptance

- Inclusion in PubMed, CAS, Scopus and Google Scholar

- Research which is freely available for redistribution

Submit your manuscript at www.biomedcentral.com/submit
C Biomed Central 